\title{
Linear Precoded Cooperative Transmission Protocol for Wireless Broadcast Channels
}

\author{
Chee Yen Leow ${ }^{* \ddagger}$, Zhiguo Ding ${ }^{\dagger}$, Kin K. Leung* \\ *Department of Electrical and Electronic Engineering, Imperial College London, SW7 2AZ, UK. \\ ${ }^{\dagger}$ Department of Communication Systems, Lancaster University, LA1 4YW, UK. \\ $\ddagger$ Faculty of Electrical Engineering,Universiti Teknologi Malaysia, 81310 Skudai, Malaysia. \\ Email:\{bruceleow, kin.leung\}@imperial.ac.uk, z.ding@lancaster.ac.uk
}

\begin{abstract}
One of the main challenges in the wireless broadcast channels is the co-channel interference among multiple destinations. Practical linear precoding techniques such as zeroforcing beamforming (ZFBF) is used to avoid the co-channel interference. However, the achievable diversity order of the ZFBF is bounded by the number of transmitter antennas. In this paper, we introduce a spectral efficient cooperative broadcast channels transmission protocol in the MISO channels using linear precoding. We evaluate the performance of the proposed protocol using the diversity and multiplexing tradeoff. The proposed protocol can achieve the maximum diversity order expressed as a sum of the transmitter antennas and the number of participating relays. For a large number of relay candidates, the diversity and multiplexing tradeoff of the proposed protocol completely surpasses the performance of the comparable scheme. Simulations have also shown the outage probability of the proposed protocol outperforms the existing scheme.
\end{abstract}

\section{INTRODUCTION}

In the wireless broadcast channels, multiple-input singleoutput (MISO) configuration is particularly of interest. In the MISO channels, the optimal sum-rate can be achieved using interference pre-substraction technique known as dirty paper coding (DPC) [1]. However, the high computation cost and complexity make it less attractive if compared to suboptimal methods such as linear precoding. An example is the zeroforcing beamforming (ZFBF) which cancels the co-channel interference among multiple users [2]. Linear precoded broadcast transmission protocol can achieve the optimal sum-rate by utilising the multi-user diversity [3] in scheduling. The ZFBF with semi-orthogonal user scheduling proposed in [4] can achieve the optimal sum-rate capacity. The merits in terms of practicality and spectral efficiency motivate the application of $\mathrm{ZFBF}$ in broadcast transmission.

On the other hand, the diversity order of the MISO broadcast channels achieved by DPC and linear precoding is constrained by the number of transmitter antennas. Increasing the number of transmitter antennas is costly due to the independent RFchain required by each additional antenna. As a practical way to improve the reliability, we propose to incorporate the cooperative transmission in the broadcast transmission. Cooperative transmission provides another dimension of spatial diversity known as cooperative diversity. The cooperative diversity is achieved by employing relay nodes to forward the information broadcast by the source [5]. No extra hardware cost is required since the relays can be formed by the idle users. Initial work focussed on single antenna node which transmits one new message only per time slot, e.g. the dynamic decode-andforward (DDF) strategy proposed in [6] which can achieve full diversity in broadcast channels. The main challenge of introducing the relay transmission in MISO broadcast channels is the co-channel interference among multiple users. This motivates the work in this paper.

In this paper, we propose a linear precoded cooperative broadcast channels (CBC) transmission protocol for MISO broadcast channels. By using the existing ZFBF with semiorthogonal user scheduling at the source [4] coupled with the ZFBF at the relay, co-channel interference among the destinations can be nullified. All qualified relays are scheduled in a round robin fashion to forward the source messages to the target destinations. At each time slot, new messages will be transmitted along with the relayed messages from the previous time slot. This non-orthogonal transmission strategy allows the source and the relays to share a common bandwidth and the outcome is a spectrally efficient cooperative transmission. At each of the destinations, simple successive decoding is used to decode the mixture of the new source message and the relayed message.

We evaluate the performance of the proposed CBC protocol using the diversity and multiplexing tradeoff [7]. The achievable diversity and multiplexing tradeoff of the proposed CBC scheme completely surpasses the non-cooperative ZFBF scheme when a large number of relays is available. The maximum diversity order expressed as a sum of number of transmitter antennas at the source and the number of available relays is achievable by the proposed scheme. Monte-Carlo simulations have also shown that the outage probability of the proposed scheme outperforms the comparable scheme.

\section{DESCRIPTION OF THE COOPERATIVE BROADCAST CHANNELS (CBC) PROTOCOL}

Consider a broadcast scenario with one source, $M$ destinations and $L$ relays. The source and relays are equipped with $M_{t}$ and $M_{r}$ antennas respectively ${ }^{1}$, while the destination nodes have single antenna. Note that $M_{r} \geq M_{t}$ and $M_{t}=M$. However, in the presentation of the proposed protocol, we set $M_{r}=M_{t}=M$ for simplicity. The relay transmission

\footnotetext{
${ }^{1}$ The assumption of multiple antennas at the relays is reasonable because multiple antennas relay can be formed by clustering several single antenna nodes. This is further supported by the fact that in wireless network, idle user set is much larger than the active user set.
} 
uses decode-and-forward (DF) strategy and the half duplex constraint is assumed. We adopt time division duplex (TDD) due to its simplicity. All channels undergo quasi static and frequency non-selective Rayleigh fading. The noise is circularly symmetric complex Gaussian distributed, $n \sim \mathcal{C N}\left(0, \sigma_{n}^{2}\right)$. The proposed CBC protocol is described in two phases, the initialization and the cooperative transmission.

\section{A. Initialization}

All nodes assume perfect knowledge of the local channel state information (CSI) to enable linear precoding at the source and relays, and coherent detection at the destinations. Using the channel reciprocity principle, the CSI can be acquired using open-loop method [8]. Using this method, the source and each destinations will take turn to broadcast the training symbols. A total of $M+1$ time slots are needed for such signaling. On the other hand, $M$ time slots are needed for the non-cooperative ZFBF broadcast scheme [4] which serves as a baseline for comparison. The CBC protocol only consumes one extra time slot. All nodes use such training information to estimate their local channels. Every relay will know whether it can decode the source messages successfully. The qualified relays will inform the destinations through the control channel. The criterion for successful decoding will be discussed in the next section.

\section{B. Cooperative Transmission}

Using user scheduling such as semi-orthogonal user selection in [4], we can have $M$ orthogonal channels, such that $h_{j} \perp h_{k}$ for any $j \neq k$. Applying the ZFBF with scheduling, we can achieve $M$ interference-free parallel transmission in a single time slot, which leads to the optimal sum-rate [4].

1) First time slot: At the first time slot, the source transmits $M$ messages simultaneously, such that $\mathbf{x}(1)=\mathbf{W} \mathbf{s}(1)$, where $\mathbf{s}(1)=\left[\begin{array}{lll}s_{1}(1) & \cdots & s_{M}(1)\end{array}\right]^{T}, s_{m}(K)$ is the message with unit energy transmitted at the $K$ th time slot to $m$ th destination. $\mathbf{W}=\left[\begin{array}{lll}\mathbf{w}_{1} & \ldots & \mathbf{w}_{M}\end{array}\right]$ is the weighting matrix. According to zero-forcing criterion [9], $\mathbf{h}_{j} \mathbf{w}_{k}=0$ for any $j \neq k$ where $\mathbf{h}_{j}$ is row vector and $\mathbf{w}_{k}$ is column vector, the weighting matrix is set to

$$
\mathbf{W}=\left[\begin{array}{ccc}
\frac{\mathbf{h}_{1}^{H}}{\left|\mathbf{h}_{1}\right|} & \cdots & \frac{\mathbf{h}_{M}^{H}}{\left|\mathbf{h}_{M}\right|}
\end{array}\right],
$$

where the normalising factor is used to ensure the energy per destination is unity, i.e. $\mathbf{W}^{H} \mathbf{W}=\mathbf{I}_{M}$. The vector $\mathbf{h}_{m}$ is the channel from source to $m$ th destination and $\left|\mathbf{h}_{m}\right|$ is used to denote the Euclidean norm of vector $\mathbf{h}_{m}$. The signal received by $m$ th destination is

$$
y_{m}(1)=\sqrt{E_{s}} \mathbf{h}_{m} \mathbf{w}_{m} s_{m}(1)+n_{m}(1),
$$

where $E_{s}$ is the average transmit energy per antenna, $n_{m}$ is the noise at $m$ th destination and $\mathbf{h}_{m} \mathbf{w}_{m}=\left|\mathbf{h}_{m}\right|$. The signal received by the $k$ th relay is

$$
\mathbf{r}_{k}(1)=\sqrt{E_{s}} \mathbf{H}_{R_{k}} \mathbf{W s}(1)+\mathbf{n}_{k}(1)
$$

where $\mathbf{H}_{R_{k}}$ is the matrix channel from source to $k$ th relay and $\mathbf{n}_{k}(1)$ is the i.i.d. noise vector at time slot 1.
Out of total $L$ available relays, there are $K$ qualified relays manage to decode the messages successfully. Since symmetrical system is considered, the sum-rate criterion is used to determine whether a relay is qualified. The criterion is written as

$$
\log _{2} \operatorname{det}\left(\mathbf{I}+\gamma_{0} \mathbf{H}_{R_{k}} \mathbf{W} \mathbf{W}^{H} \mathbf{H}_{R_{k}}^{H}\right)>M R
$$

where $R$ is the target rate in bits $/ \mathrm{s} / \mathrm{Hz}$ for each destination and $\gamma_{0}=\frac{E_{s}}{\sigma_{n}^{2}}$ denotes the mean SNR. All $K$ qualified relays decode the messages and store them in memory.

2) Second time slot: During the second time slot, the source will transmit $M$ new messages concurrently, such that $\mathbf{x}(2)=\mathbf{W} \mathbf{s}(2)$. Denote row vector $\mathbf{g}_{R_{k}, m}$ as the channel between the relay $R_{k}$ and the $m$ th destination. All $K$ qualified relays are scheduled to transmit in a round robin fashion. Only one relay will transmit at each cooperative time slot. Specifically, the first relay will forward the messages $\mathbf{s}(1)$ with precoding as $\mathbf{x}_{R_{1}}=\mathbf{P}_{R_{1}} \mathbf{s}(1)$, where the precoding matrix $\mathbf{P}_{R_{1}}=\left[\mathbf{p}_{R_{1}, 1} \cdots \mathbf{p}_{R_{1}, m} \cdots \mathbf{p}_{R_{1}, M}\right]$, each column vector $\mathbf{p}_{R_{1}, m}$, for $m=1, \ldots, M$ should ensure no interference to other destinations. Denote $\mathbf{A}$ as a matrix which contains all the interfering channels (all channels other than its targeted destination). In this instance, denote

$$
\mathbf{A}=\left[\begin{array}{llllll}
\mathbf{g}_{R_{1}, 1}^{T} & \cdots & \mathbf{g}_{R_{1}, m-1}^{T} & \mathbf{g}_{R_{1}, m+1}^{T} & \cdots & \mathbf{g}_{R_{1}, M}^{T}
\end{array}\right]^{T},
$$

and we set $\mathbf{p}_{R_{1}, m}$ as

$$
\mathbf{p}_{R_{1}, m} \in \text { Null Space of } \mathbf{A},
$$

so that $\mathbf{g}_{R_{1}, i} \mathbf{p}_{R_{1}, j}=0$ for any $i \neq j$. The computation of the null space can be found in [10]. The energy of $\mathbf{p}_{R_{1}, m}$ is unity, i.e $\mathbf{p}_{R_{1}, m}^{H} \mathbf{p}_{R_{1}, m}=1$. The null space precoding well known as ZFBF is able to multiplex $M$ stream of messages simultaneously and eliminate the interference among destinations. At the $m$ th destination, the observation is

$y_{m}(2)=\sqrt{E_{s}}\left|\mathbf{h}_{m}\right| s_{m}(2)+\sqrt{E_{s}} \mathbf{g}_{R_{1}, m} \mathbf{p}_{R_{1}, m} s_{m}(1)+n_{m}(2)$.

Although the remaining relays also receive a mixture of the messages, they will be able to decode the source messages using simple successive decoding since they satisfy the qualification criterion in eq. 4.

3) Continue until all $K$ qualified relays have been used: The relaying process will continue until all $K$ qualified relays have been used. Let $\mathbf{s}=\left[\begin{array}{lll}s_{m}(1) & \cdots & s_{m}(K+1)\end{array}\right]^{T}$,

$$
\mathbf{H}_{m}=\left[\begin{array}{cccc}
\left|\mathbf{h}_{m}\right| & 0 & \cdots & 0 \\
\mathbf{g}_{R_{1}, m} \mathbf{p}_{R_{1}, m} & \left|\mathbf{h}_{m}\right| & \cdots & 0 \\
\vdots & \ddots & \ddots & \vdots \\
0 & \cdots & \mathbf{g}_{R_{K}, m} \mathbf{p}_{R_{K}, m} & \left|\mathbf{h}_{m}\right|
\end{array}\right],
$$

and $\mathbf{n}=\left[\begin{array}{lll}n_{m}(1) & \cdots & n_{m}(K+1)\end{array}\right]^{T}$, the signal model for $K+1$ time slots is

$$
\mathbf{y}_{m}=\mathbf{H}_{m} \mathbf{s}+\mathbf{n} \text {. }
$$

The mutual information at node $m$ for $K+1$ time slots is

$$
\mathcal{I}_{K}=\frac{1}{K+1} \log _{2} \operatorname{det}\left(\mathbf{I}_{K+1}+\gamma_{0} \mathbf{H}_{m} \mathbf{H}_{m}^{H}\right)
$$


where $\gamma_{0}=\frac{E_{s}}{\sigma_{n}^{2}}$ and the subscript $\mathrm{K}$ of $\mathcal{I}_{K}$ denotes the total number of relays used in the cooperative transmission.

\section{Diversity and Multiplexing tradeoff Analysis}

This section provides the performance evaluation of the proposed $\mathrm{CBC}$ protocol using the diversity and multiplexing tradeoff. Several key questions such as the probability that a relay is qualified, number of qualified relays and outage probability are derived. Using the outage probability upper bound, the diversity and multiplexing tradeoff is obtained.

First, we wish to determine the probability that a relay will decode the source information successfully. In order to formalise the qualification criterion into probability, we determine the statistical distribution of the SNR of each relay. We generalise the channel matrix from the source with $M_{t}$ transmitter, to the relay with $M_{r}$ receiver, as $\mathbf{H}_{w}$ with dimension $M_{r} \times M_{t}$. Recall equation 3, the virtual channel matrix can be written as $\mathbf{H}=\mathbf{H}_{w} \mathbf{W}$ where $\mathbf{H}_{w} \sim \mathcal{C N}\left(0, \mathbf{I}_{M_{t}} \otimes \boldsymbol{\Sigma}\right)$, $\Sigma$ is the covariance matrix with dimension $M_{r} \times M_{r}$. The weighting matrix $\mathbf{W}$ is treated as a constant matrix with orthogonal columns, i.e $\mathbf{w}_{i}^{H} \mathbf{w}_{j}=0$ when $i \neq j, i, j$ are the column numbers. This is valid when forced semi-orthogonality user scheduling proposed by [4] is employed. In this case, the columns of $\mathbf{H}$ remain independent of each other, i.e $\mathbf{h}_{i}^{H} \mathbf{h}_{j}=0$ when $i \neq j, i, j$ are the column numbers. Using the zero-forcing criterion at the receiver, we can decompose the SNR into $M_{r}$ parallel streams. Denote $\mathbf{h}_{k}$ as the $k$ th column of $\mathbf{H}$ and $\tilde{\mathbf{H}}$ as the remaining columns of $\mathbf{H}$ after removing the $k$ th column while $\mathbf{w}_{k}$ is the $k$ th column of $\mathbf{W}$. The SNR of $k$ th stream can be obtained using the following lemma.

Lemma 1: The SNR of $k$ th stream can be expressed as

$$
\gamma_{k}=\gamma_{o} \mathbf{h}_{k}^{H} \mathbf{Q} \Lambda \mathbf{Q}^{H} \mathbf{h}_{k},
$$

where $\gamma_{0}=\frac{E_{s}}{\sigma_{n}^{2}}$ and $\mathbf{Q} \boldsymbol{\Lambda} \mathbf{Q}^{\mathbf{H}}=\mathbf{I}-\tilde{\mathbf{H}}\left(\tilde{\mathbf{H}}^{H} \tilde{\mathbf{H}}\right)^{-1} \tilde{\mathbf{H}}^{H}$. Note that $\mathbf{Q}$ is unitary matrix containing the eigen vectors, $\boldsymbol{\Lambda}$ is diagonal matrix containing the eigen values.

Proof: Refer [11].

Before determining the distribution of the SNR of $k$ th stream, we need to know the distribution of $\mathbf{h}_{k}$. We know that $\mathbf{h}_{k}$ is the weighted sum of complex Gaussian vectors. The subsequent lemma provides us the distribution of $\mathbf{h}_{k}$.

Lemma 2: Given $\mathbf{H}_{w} \sim \mathcal{C N}\left(0, \mathbf{I}_{M_{t}} \otimes \boldsymbol{\Sigma}\right), \mathbf{h}_{k}=\mathbf{H}_{w} \mathbf{w}_{k}$ is complex Gaussian distributed with zero mean and covariance $\left|\mathbf{w}_{k}\right|^{2} \Sigma$, such that $\mathbf{h}_{k} \sim \mathcal{C N}_{M r}\left(0,\left|\mathbf{w}_{k}\right|^{2} \Sigma\right)$.

Proof: Refer [12].

By using lemma 1 and lemma 2, the distribution of the $k$ th stream SNR at the relay receiver is obtained, as shown in the following theorem.

Theorem 1: When $\mathbf{H}_{w} \sim \mathcal{C N}\left(0, \mathbf{I}_{M_{t}} \otimes \sigma^{2} \mathbf{I}_{M r}\right)$, the SNR of the $k$ th stream at the relay receiver is a chi-square variable with probability density function (PDF)

$$
\begin{aligned}
f\left(\gamma_{k}\right)= & \frac{\left(\gamma_{k}\right)^{M_{r}-M_{t}}}{\left(\gamma_{0} \sigma^{2}\left|\mathbf{w}_{k}\right|^{2}\right)^{\left(M_{r}-M_{t}+1\right)}\left(M r-M_{t}\right) !} \\
& \times \exp \left(-\frac{\gamma_{k}}{\gamma_{0} \sigma^{2}\left|\mathbf{w}_{k}\right|^{2}}\right) .
\end{aligned}
$$

The corresponding cumulative density function (CDF) is

$$
F\left(\gamma_{k}\right)=1-\exp \left(-\frac{\gamma_{k}}{\gamma_{0} \sigma^{2}\left|\mathbf{w}_{k}\right|^{2}}\right) \sum_{n=0}^{M_{r}-M_{t}} \frac{1}{n !}\left(\frac{\gamma_{k}}{\gamma_{0} \sigma^{2}\left|\mathbf{w}_{k}\right|^{2}}\right)^{n} .
$$

Proof: Refer [12].

With the knowledge of the distribution of the $k$ th stream SNR, we can determine the probability that a relay is qualified. Assuming the SNR of each stream is independent, we obtain the following lemma.

Lemma 3: By assuming quasi static and frequency nonselective Rayleigh fading channels, at high SNR, the probability that a relay is qualified can be approximated as

$$
P(A) \approx 1-M_{r} \frac{\gamma^{M_{r}-M_{t}+1}}{\left(M_{r}-M_{t}+1\right) !},
$$

where A is the event that a relay is qualified and $\gamma=\frac{2^{R}-1}{\gamma_{0}\left|\mathbf{w}_{k}\right|^{2}}$.

Proof: Refer [12]. Note that the outage event of each stream in linear receiver is not strictly independent. However, the independent SNR assumption does not affect the diversity of the system and is used to make the analysis tractable, as discussed in [13].

Given total $L$ available relays, we are able to determine the number of qualified relays using the following theorem. Note that we use special symbol $\doteq$ to denote the exponential equality, i.e. $f\left(\gamma_{0}\right) \doteq \gamma_{0}^{n}$ to denote $\lim _{\gamma_{0} \rightarrow \infty} \frac{\log _{2} f\left(\gamma_{0}\right)}{\log _{2} \gamma_{0}}=n$.

Theorem 2: By assuming quasi static and frequency nonselective Rayleigh fading channels, the size of the qualified relay set $\left\{i: \operatorname{det}\left(\mathbf{I}+\gamma_{0} \mathbf{H}_{R_{i}} \mathbf{W} \mathbf{W}^{H} \mathbf{H}_{R_{i}}^{H}\right) \geq 2^{M_{r} R}\right\}$ at high SNR can be approximated as

$$
\begin{aligned}
P(K) & \approx \frac{L !}{(L-K) ! K !}\left(M_{r} \frac{\gamma^{M_{r}-M_{t}+1}}{\left(M_{r}-M_{t}+1\right) !}\right)^{L-K} \\
& \doteq \gamma_{0}^{-\left(M_{r}-M_{t}+1\right)(L-K)(1-r)},
\end{aligned}
$$

where $\gamma=\frac{2^{R}-1}{\gamma_{0}\left|\mathbf{w}_{k}\right|^{2}}, K$ is the number of qualified relays, $L$ is the number of available relays and $K \leq L$. Note that $r$ is defined in eq. 18. As $\gamma_{0} \rightarrow \infty, P(K=L) \rightarrow 1$ and the probability for $K \neq L$ is very small such that $P(K \neq L) \rightarrow 0$. Proof: Refer [12].

Knowing the number of qualified relays, we proceed to determine the outage performance of the proposed protocol. Define the outage event for the proposed CBC protocol as

$$
\mathcal{O} \triangleq \bigcup_{\mathrm{K}} \mathcal{O}_{K}
$$

where $\mathcal{O}_{K}$ is the event that the mutual information when there are $K$ qualified relays is below the target data rate, i.e $\mathcal{I}_{K} \leq R$. The outage probability of the system can be expressed as

$$
P(\mathcal{O})=\sum_{K=0}^{k} P\left(\mathcal{O}_{K}\right) P(K),
$$

where $P(K)$ is stated in theorem 2 and $P\left(\mathcal{O}_{\mathrm{K}}\right)$ is the outage probability when $K$ relays are qualified. The upper bound of $P\left(\mathcal{O}_{\mathrm{K}}\right)$ is expressed in the following theorem. 
Theorem 3: By assuming quasi static and frequency nonselective Rayleigh fading channels, the outage probability for the event that $K$ relays are qualified at high SNR can be expressed as

$$
\begin{aligned}
P\left(\mathcal{O}_{\mathrm{K}}\right) \leq & \left((-1)^{K}-\gamma_{0}^{(K+1) r} \sum_{i=0}^{K-1} \frac{\left(\ln \gamma_{0}^{(K+1) r}\right)^{i}}{i !(-1)^{K-i}}\right) \\
& \times \frac{\left(\frac{\gamma_{0}^{r}-1}{\gamma_{0}}\right)^{M_{t}}}{M_{t} ! \gamma_{0}^{K}} \doteq \gamma_{0}^{-\left[M_{t}(1-r)+K-(K+1) r\right]} .
\end{aligned}
$$

Proof: Refer [12].

Recall that the diversity and multiplexing gain can be defined as [7]

$$
d \triangleq \lim _{\gamma_{0} \rightarrow \infty}-\frac{\log \left[P_{e}\left(\gamma_{0}\right)\right]}{\log \gamma_{0}} \text { and } r \triangleq \lim _{\gamma_{0} \rightarrow \infty} \frac{R\left(\gamma_{0}\right)}{\log \gamma_{0}}
$$

where $P_{e}$ is maximum likelihood (ML) probability of detection error and $R$ is the data rate in bits/Hz/s. Since the ML error probability can be tightly bounded by the outage probability at high SNR, the outage probability will be used. Combining the results from theorem 2 and 3 , the following diversity multiplexing tradeoff for the proposed $\mathrm{CBC}$ protocol is obtained.

Theorem 4: By assuming quasi-static and frequency nonselective Rayleigh fading channels, the achievable diversitymultiplexing tradeoff for the proposed $\mathrm{CBC}$ protocol at high SNR is

$$
d(r)=M_{t}(1-r)+[L-(L+1) r]^{+},
$$

where $0 \leq r \leq 1$ and $(x)^{+}$denotes $\max \{x, 0\}$.

As a comparison, the optimal diversity and multiplexing tradeoff of the non-cooperative ZFBF broadcast scheme can be written as

$$
d_{Z F B F}(r)=M_{t}(1-r),
$$

where $0 \leq r \leq 1$.

For a fixed data rate, the diversity order of the noncooperative ZFBF scheme is constrained by the number of transmitter antennas $M_{t}$ at the source while the proposed CBC protocol can achieve the maximum diversity order of $M_{t}+L$. It provides extra $L$ diversity order which is a function of number of participating relays. The full diversity gain can be achieved since all qualified relays are scheduled in a round robin fashion to assist all destinations. When $r \leq \frac{L}{L+1}$, the diversity order of $\mathrm{CBC}$ is always greater or equal to the diversity order of non-cooperative ZFBF,i.e. $d(r) \geq d_{Z F B F}(r)$. When $L$ is large enough, i.e $\frac{L}{L+1} \approx 1$, the achievable diversity and multiplexing tradeoff of the proposed $\mathrm{CBC}$ protocol is always greater than the non-cooperative ZFBF tradeoff, i.e. $d(r)>d_{Z F B F}(r)$. This condition can be easily satisfied in real wireless network, where there are more idle users than active users. The great performance improvement in term of diversity and multiplexing tradeoff justified the contribution of the proposed $\mathrm{CBC}$ protocol.

Fig. 1 shows the diversity and multiplexing tradeoff of the non-cooperative ZFBF scheme and the proposed protocol with

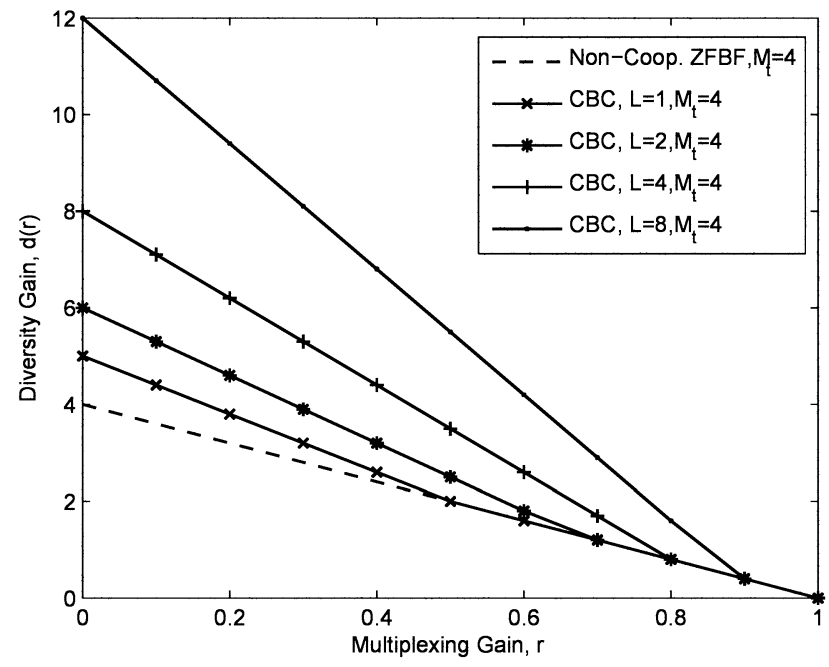

Fig. 1. Diversity and multiplexing tradeoff of the non-cooperative ZFBF scheme and the proposed $\mathrm{CBC}$ scheme with various number of available relays, $L$

various $L$ and fixed $M_{r}=M_{t}=4$. The diversity order of the proposed protocol is always higher than the non-cooperative ZFBF scheme. The proposed protocol has a flexible diversity order expressed as a sum of the number of transmitter antennas and the number of relays while the non-cooperative ZFBF scheme has fixed diversity order limited by the number of transmitter antennas. As the number of relays increases, the diversity and multiplexing tradeoff of the proposed protocol improves and approaches the MISO upper bound.

\section{Numerical RESUlts}

In this section, we present the numerical results to demonstrate the performance gain of the proposed CBC scheme in comparison with the existing non-cooperative ZFBF scheme. Two experiments are carried out using Monte-Carlo simulation to compare the performance of the $\mathrm{CBC}$ scheme with the noncooperative ZFBF. In the simulations, the number of scheduled user in one time slot, $M$ is equal to number of the transmitter antennas, $M_{t}$ and all available relays are used, i.e. $K=L$, as stated in theorem 2.

In the first experiment, the outage probability of the proposed protocol and the non-cooperative ZFBF scheme is plotted as a function of SNR, as in fig. 2. The target data rate $R$ is varied from 1 to $6 \mathrm{bits} / \mathrm{s} / \mathrm{Hz}$. The following parameters are fixed. The number of source antennas and relay antennas, $M_{t}=M_{r}=2$ and the number of available relays $L=2$. The outage performance of the proposed protocol is always better than the non-cooperative ZFBF scheme regardless of the target data rate. The performance gain of the proposed protocol is a direct result of exploiting the relays to achieve another dimension of diversity gain. As the data rate decreases, the outage probability of both schemes improve. This fits the general understanding of the fixed rate communication, transmitting with lower target data rate provides more reliable transmission but sacrifices the throughput.

In the second experiment, the outage probability versus SNR of the non-cooperative ZFBF scheme and the proposed CBC 


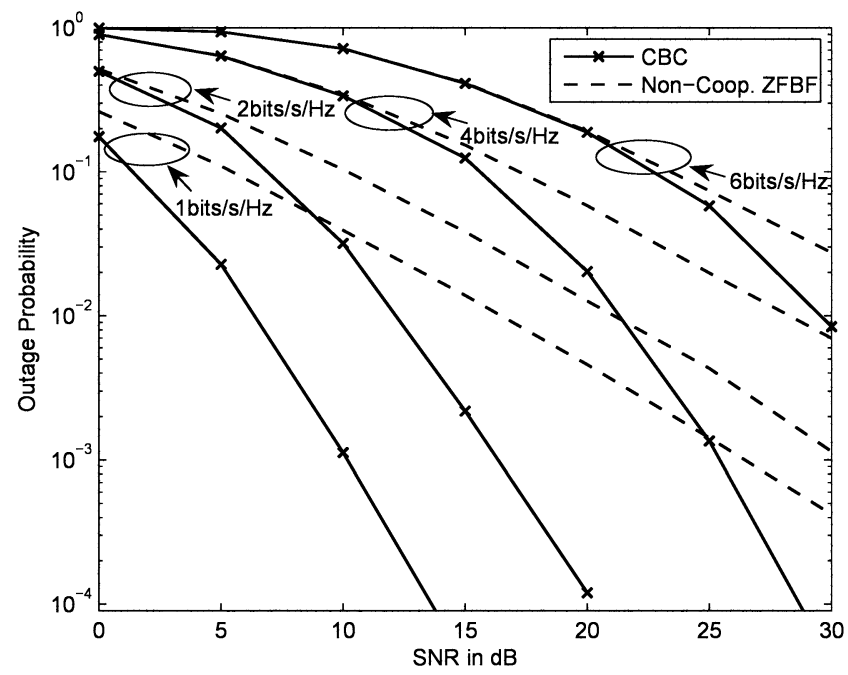

Fig. 2. Outage probability versus SNR of the non-cooperative ZFBF scheme and the proposed $\mathrm{CBC}$ scheme with various target data rate, $R$.

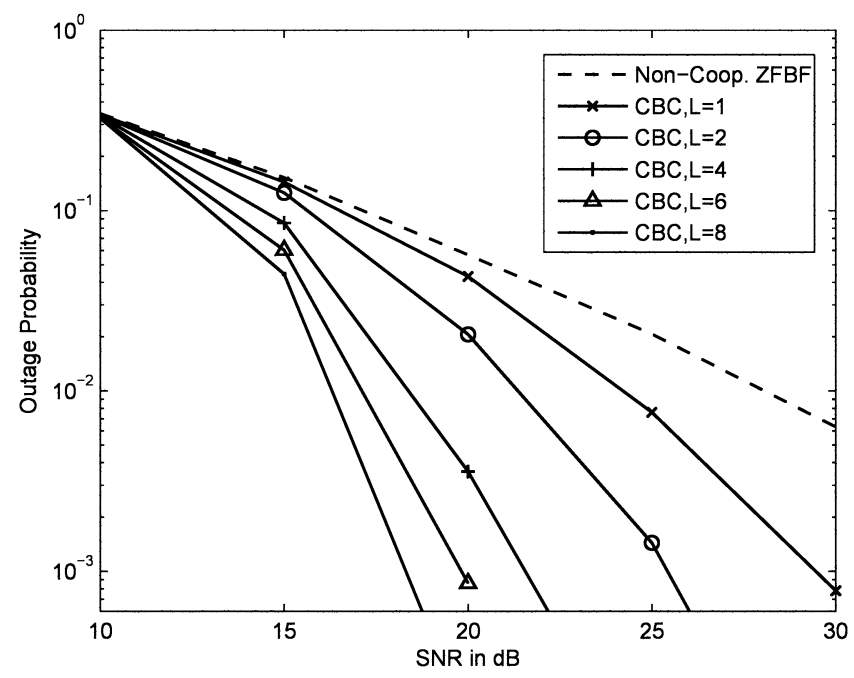

Fig. 3. Outage probability versus SNR of the non-cooperative ZFBF scheme and the proposed CBC scheme with various number of available relays, $L$.

scheme with different number of available relays are simulated to study the relationship between the number of relays and the outage performance. We fixed $M_{t}=M_{r}=2$ and $R=4$ bits/s/Hz. $L$ is varied from 1 to 8 . Refer to fig. 3 , with only one relay, the outage probability of proposed $\mathrm{CBC}$ protocol is smaller than the non-cooperative ZFBF scheme. As we increase the number of available relays, the outage probability of the proposed scheme decreases. This implies that increasing the relays will improve the diversity order of the protocol. Note that the diversity order depends on the slope of the outage probability curve. The higher the diversity order, the steeper the slope is. The result demonstrates the reliability of the broadcast transmission can be improved by adding relays without incurring additional hardware cost. The number of relays can be varied in a flexible way. For instance, during bad channel condition, more relays should be made available to cooperate while the reverse during good channel condition.

\section{CONClusion}

A spectrally efficient linear precoded cooperative broadcast channels transmission protocol is proposed. The proposed protocol rules out the co-channel interference among multiple destinations using the zero-forcing beamforming. The outage behaviour and the diversity and multiplexing tradeoff of the proposed protocol is studied in order to quantify its performance. A maximum diversity order expressed as a sum of the number of transmitter antennas and the available relays can be achieved. When the number of available relays is large, the diversity and multiplexing tradeoff curve of the proposed CBC protocol completely outperforms the existing non-cooperative ZFBF scheme. The Monte-Carlo simulations also proved that the proposed $\mathrm{CBC}$ protocol achieves better system robustness than the comparable scheme.

\section{ACKNOWLEDGEMENT}

This research was sponsored by US Army Research laboratory and the UK Ministry of Defence and was accomplished under Agreement Number W911NF-06-3-0001. The views and conclusions contained in this document are those of the authors and should not be interpreted as representing the official policies, either expressed or implied, of the US Army Research Laboratory, the U.S. Government, the UK Ministry of Defense, or the UK Government. The US and UK Governments are authorized to reproduce and distribute reprints for Government purposes notwithstanding any copyright notation hereon.

\section{REFERENCES}

[1] M. Costa, "Writing on dirty paper (corresp.)," IEEE Transaction of Information Theory, vol. 29, no. 3, pp. 439-441, May 1983.

[2] A. Wiesel, Y. Eldar, and S. Shamai, "Zero-forcing precoding and generalized inverses," IEEE Transactions on Signal Processing, vol. 56, no. 9, pp. 4409-4418, Sep 2008.

[3] D. Tse and P. Viswanath, Fundamentals of Wireless Communication. New York: Cambridge University Press, 2005.

[4] T. Yoo and A. Goldsmith, "On the optimality of multiantenna broadcast scheduling using zero-forcing beamforming," IEEE Transactions on Information Theory, vol. 24, no. 3, pp. 528-541, Mar. 2006.

[5] J. Laneman, D. Tse, and G. Wornell, "Cooperative diversity in wireless networks: Efficient protocols and outage behavior," IEEE Transactions on Information Theory, vol. 50, no. 12, pp. 3062-3080, Dec. 2004.

[6] K. Azarian, H. El Gamal, and P. Schniter, "On the achievable diversitymultiplexing tradeoff in half-duplex cooperative channels," IEEE Transactions On Information Theory, vol. 51, no. 12, pp. 4152-4172, 2005.

[7] L. Zheng and D. Tse, "Diversity and multiplexing: A fundamental tradeoff in multiple-antenna channels," IEEE Transactions on Information Theory, vol. 49, no. 5, pp. 1073-1096, May 2003.

[8] E. Biglieri, R. Calderbank, A. Constantinides, A. Goldsmith, A. Paulraj, and H. V. Poor, MIMO Wireless Communications. Cambridge: University Press, Cambridge, 2007.

[9] A. Paulraj, R. Nabar, and D. Gore., Introduction to Space-Time Wireless Communications. Cambridge: University Press, Cambridge, 2003.

[10] G. Strang, Linear Algebra and Its Applications, 3rd ed. New York: Thomson Learning, 1988.

[11] M. Rupp, C. Mecklenbrauker, and G. Gritsch, "High diversity with simple space time block-codes and linear receivers," in Proc. IEEE Global Telecommunications Conference GLOBECOM '03, vol. 1, 2003, pp. 302-306.

[12] C. Y. Leow, Z. Ding, and K. K. Leung, "Linear precoded cooperative transmission protocol for wireless broadcast channel," Submitted to IEEE Transactions on Wireless Communications,

[13] A. Hedayat and A. Nosratinia," "Outage and diversity of linear receivers in flat-fading mimo channels," IEEE Transactions on Signal Processing, vol. 55, no. 12, pp. 5868-5873, Dec. 2007. 